\title{
A REVIEW ON CHARACTERIZATION OF SOLID DISPERSION
}

\author{
Vishakha Sharma \\ Department of Pharmacy \\ M.J.P Rohilkhand University, \\ Bareilly, Uttar Pradesh, India
}

\begin{abstract}
Solid dispersion is a two component system or it can be defined as the drug-polymer interaction. Basically, this system is confined in order to increase the solubility of poor water soluble drugs by making the drug dispersed in a polymer. It is based on the concept that the drug (hydrophobic) is dispersed in an inert water-soluble carrier or polymer (hydrophilic) at solid state. In this review, we summarize our current understanding of solid dispersions by studying its characteristics ${ }^{1}$.
\end{abstract}

Keywords - solid dispersion, scanning electron microscopy, XRD, SEM, FTIR, DSC

\section{INTRODUCTION}

Solid dispersion is a science of dispersing or a technology in which we disperse a solid matrix into a polymer matrix or a liquid one ${ }^{2}$. This has been widely used in pharmaceutical industries as it has become a useful criterion in bringing up the solubility of poorly soluble drugs. Generally solubility, stability and dissolution problems are associated with many pharmaceutical formulations ${ }^{3}$. With the upbringing of solid dispersion, the problems associated with the poorly soluble drugs have been diminished to a larger extent. This has not only led the enhanced solubility but also led to the increased dissolution rate and bioavailability of poorly soluble drugs, which in turn will promote the use of poorly, water soluble drugs in pharmaceutical formulation of solid dispersion ${ }^{4}$.

\section{CHARACTERIZATION}

Characterization methods are generally employed for differentiating between solid solutions and solid dispersions. Some of the methods are described here:

\section{Differential scanning calorimetry (DSC)}

It is an analytical technique in which the difference in the amount of heat required to increase the temperature of a sample and reference is measured. With the help of DSC, we can find the melting temperatures ${ }^{5}$. DSC helps to study the thermal behaviour of various substances. Generally, the interaction between the drug and the polymer causes change in exothermic and endothermic peaks ${ }^{6}$. According to the different processing conditions, different thermograms were observed. Mainly, DSC is used in polymer research for three different types of experiments:

a) glass-rubber transition temperature ( $\mathrm{Tg}$ value) determination.

b) Melting temperature and heat $(\mathrm{Tm} / \mathrm{Tc}$ - value and $\mathrm{Hf} / \mathrm{Hc}-$ value) determination.

c) Cure measurements or reacting systems measurements ${ }^{7}$.

Nuclear magnetic resonance (NMR)

To study solvates, polymorphs, provide quantitative data in both drug substance and drug product and study complicated formulations such as amorphous dispersion can be well studied by nuclear magnetic resonance ${ }^{8}$. It is used to investigate the polymorphism by probing the atoms in the solid state. The changes in chemical shift can be connected with the change in conformation or chemical nature of the compound ${ }^{9}$.

\section{Scanning electron microscopy (SEM)}

The shape, morphology, properties of drug crystals like particle size and chemical composition can be explained through the range of parameters obtained by SEM. Scanning electron microscopy (SEM) is widely used in the characterization of amorphous solid dispersions ${ }^{10}$.

\section{Thermal gravimetric analysis (TGA)}

It is a powerful technique for studying the changes in weight of a sample when heated, cooled or when held at constant temperature. It is used to determine temperature in which drug decay occurs. It can also be used to determine the moisture content in solid dispersion and to measure the weight loss at different temperatures which provides information about the thermal stability ${ }^{11}$.

\section{Powder x-ray diffraction}

It can be used to determine the crystalline phase in a mixed system. The crystallinity phase gives sharp narrow diffraction peaks and the amorphous component gives a broad peak. However, too much crystallinity results in brittleness. It can also be used to screen physical stability, to characterize new forms, to screen crystal and amorphous dispersions. 


\section{International Journal of Engineering Applied Sciences and Technology, 2019 \\ Vol. 4, Issue 6, ISSN No. 2455-2143, Pages 127-128 \\ Published Online October 2019 in IJEAST (http://www.ijeast.com)}

\section{Fourier transformed infrared spectroscopy}

FTIR takes into account the specific absorbance of molecular vibrations for quality assessment. It offers molecular and structural conformation in the solid state by probing vibration of atoms. It can be used to determine the changes in bonding between functional groups, to detect chemical properties and to detect the crystallinity range from 1 to $99 \%$ in pure material ${ }^{12}$.

\section{CONCLUSION}

We can improve the solubility of poorly soluble drugs with enhanced bioavailability by the technique of solid dispersion. All the above characteristics or parameters played an important role in formulation of an ideal solid dispersion.

\section{REFERENCE}

[1] Singh S., Baghel RS., Yadav LA. (2011) Review on Solid Dispersion: International Journal of Pharmacy and Life Sciences 2011; 2(9): (pp.1078-1095).

[2] Serajuddin A. (1999). Solid Dispersion Technique, Journal of Pharmaceutical Sciences, 1999; 88(10): (pp.891-900).

[3] Lipinski CA. (2000). Drug-like properties and The Causes of Poor Solubility and Poor Permeability, Journal of Pharmacology and Toxicology. Methods 2000; 44: (pp.235-249).

[4] Aggarwal S., Goel A. (2012), Solubility and Its Enhnacement Techniques of Poorly Soluble Drugs. Int. Journal of Universal Pharmacy and Life Sciences 2012; 2(1): (pp.65-82).

[5] Leuner C, Dressman J. (2000) Improving drug solubility for oral delivery using solid dispersions. Eur. J. Pharm. 2000; 50: (pp. 47-60).

[6] Jain RK, Sharma DK, Jain S, Kumar S and Dua JS. (2006) Studies on Solid Dispersion of Nimesulide with Pregelatinized Starch. Biosciences, biotechnology research Asia 2006, 3(1a): 3(pp.151-153).

[7] Kakumanu VK, Bansal AK. (2003) Supercritical Fluid Technology in Pharmaceutical Research. CRIPS. 2003; 4(83): (pp. 8- 12).

[8] Narang A, Shrivastava A. (2002) Drug Dev. Ind. Pharm. 2002; 26(8): (pp.111-115).

[9] Patidar K, Kshirsagar MD. (2011) Solid Dispersion Technology: A Boon for Poorly Water Soluble Drugs. Indian Journal of Novel Drug Delivery 2011; 3(2): (pp. 83-90).

[10] Pinnamaneni S, Das NG, Das SK. (2002), Formulation Approaches for Orally Administered Poorly Soluble Drugs. Pharma. 2002; 57: (pp.291-300).

[11] Habib MJ. (2000), Pharmaceutical Solid Dispersion Technology. Washington: CRC; 2000. (pp. 35-78).
[12] Kumar Ashwini G., Choudhary Kumar Ram, Chaitanya CH.(2011), Asian Journal of Pharmaceutical and Clinical Research, Volume 4, Issue 2, 2011(pp.- 36). 